\title{
Hacia una visión holística de la educación universitaria: el amor y el caos un proceso creativo para el aprendizaje
}

\author{
Ileana Castillo Cedeño ${ }^{1 *}$ \\ Centro de Investigación y Docencia en \\ Educación de la Universidad Nacional \\ Heredia, Costa Rica \\ Claudia Marín Gutiérrez ${ }^{2 *}$ \\ Programa de Evaluación Académica y Desarrollo Profesional de la Universidad Nacional \\ Heredia, Costa Rica
}

Recibido 2 de setiembre de 2008 • Aprobado 23 de marzo de 2009

\begin{abstract}
Resumen. En este ensayo se plantea que si la educación superior quiere trascender, es necesario que desde ella se geste la idea de que el amor es el referente fundamental de la vida, y que es necesario potenciar esa esencia, más allá de lo externo y lo aparente o de temáticas aisladas dentro de planes de estudio que, muchas veces, se quedan en discursos y propuestas, y que lejos de germinar humanidad, llevan a su exterminio. Implica una visión de Amor universal que concibe el arte, como forma de expresión y de encuentro.
\end{abstract}

Palabras clave. Educación superior, holismo, amor, incertidumbre, caos, creatividad.

Abstract. In this essay it is stated that for superior education to transcend, it must develop from within the idea that love is the fundamental reference of life, and it must potentiate this essence, beyond what is external and apparent and beyond the isolated subjects of the curricula, which many times are no more than words and proposals on paper, which far from germinating humanity, lead to its extinction. It involves a vision of Universal Love that considers art as a way of expression and encounter.

Key words. Superior education, holistic, love, not knowing, caos, creativity.

\footnotetext{
Doctora en Mediación Pedagógica. Su formación inicial fue en educación preescolar. Máster en Psicopedagogía. Egresada de Doctorado en Diseño Curricular y Evaluación Educativa de la Universidad de Valladolid, España. Especialista en didáctica de la Educación del consumidor. Ha laborado en distintos niveles del sistema educativo, desde preescolar hasta el universitario y diversas poblaciones, entre las que destacan: adolescentes de comunidades en desventaja social y adultos mayores. Actualmente, se desempeña como Vicedecana del Centro de Investigación y Docencia en Educación, Heredia, Costa Rica. Correo electrónico: icastill@una.ac.cr
}

2 Especialidad en Mediación Pedagógica. Formación inicial en Educación Preescolar. Egresada de la Licenciatura en Arte Escénico de la Universidad Nacional, Costa Rica. Máster en Educación. Miembro fundador de la Asociación de Mujeres Universitarias de Heredia, Costa Rica. Ha desempeñado funciones como docente en los niveles de educación infantil, atención integral de infantes y superior, así como grupos de población en riesgo psicosocial. Directora de Cen-Cinai Ministerio de Salud, Heredia, Costa Rica. Académica en la formación de estudios de Preescolar. Cuenta con varios premios en el área de las letras. En la actualidad, forma parte del equipo de trabajo de la Dirección de Docencia de la Universidad Nacional, Costa Rica, como académica en el Programa de Evaluación Académica y Desarrollo Profesional.

Correo electrónico: $\underline{\text { cmar@una.ac.cr }}$

* El nombre de las autoras aparece en orden alfabético por apellido. 
El cambio en la visión de lo que significa la educación superior, necesariamente, tiene que ver con una mirada comprensiva, compasiva y sobre todo, comprometida, con los cambios históricos, sociales, culturales, económicos, políticos, éticos que ocurren en nuestras sociedades, los que nos deben llevar a pensar en que los procesos de transformación global, requieren, tal como lo plantea Boff ( 2002), nuevas formas de acercarse a la realidad, pensarla, sentirla y actuar, en coherencia con esas nuevas demandas, vinculadas no sólo con las ofertas de mercado de las sociedades capitalistas, sino, y principalmente, ligadas a una nueva conciencia planetaria, que establece como principio básico la VIDA.

Si la médula del proceso educativo, es la formación integral del ser humano, es coherente reflexionar y actuar, en función de la articulación de saberes, sentires y placeres. Toda pedagogía liberadora, tiene que interconectarse, inevitablemente, con el $A M O R$, lo que involucra una carga inmensa de sentido, ligado a la creatividad y a la espiritualidad lo que, a su vez, nos lleva a pensar en que ésta es una tarea urgente de la educación: el trascender el discurso en función del ser.

En la actualidad, cada vez más personas reconocen que si no ubicamos el amor y la espiritualidad como el centro de la transformación humana, probablemente, como especie pronto dejaremos de existir. Maturana (1999), señala que somos "animales amorosos", por tanto, debemos comprender que la esencia de todas las cosas está en el AMOR.

Según Morin (2003): "Nosotros, vivientes y por consiguiente humanos, hijos de las aguas, de la tierra y del sol, somos una pequeña paja, un feto incluso, de la diáspora cósmica, unas cuantas migajas de la existencia solar, un menudo brote de la existencia terrena” (p. 29).

Como partícula cósmica, nos vemos sorprendidos y llamados por esa mágica energía que todo lo transforma e interconecta. Si la educación superior quiere repercutir en la vida, es necesario gestar ese sentimiento de amor, como referente fundamental para la supervivencia. Es necesario y urgente, potenciar ese vínculo, más allá de lo externo y lo aparente o de temáticas aisladas dentro de planes de estudio, que, muchas veces, se quedan en discursos y propuestas que lejos de germinar humanidad, llevan a su exterminio.

Desde este punto de vista, las universidades del siglo XXI, y en particular, la universidad denominada Necesaria, tiene muchos retos que enfrentar, uno de los principales, es el reconocimiento de la diversidad, en todas sus expresiones y manifestaciones. Vale la pena señalar, que pese a las muchas resistencias que puedan encontrarse en el camino para darle una dimensión más holística a la educación superior, se hacen esfuerzos desde el nivel central de la administración, hasta cada una de las Facultades, Centros y Sedes, que ponen en evidencia, que se ha iniciado un proceso de resignificación del quehacer universitario, en el que se refleja una preocupación y compromiso con la vida.

Ese compromiso encierra esa mirada de amor universal, que sobrepasa el ámbito de lo individual para resaltar la solidaridad y la cooperación; implica conocimiento de que como seres vivos, crecemos y desplegamos todo nuestro potencial a partir de la interconexión con otras existencias, es ese proceso de interdependencia, lo que le da sentido a la vida y la sitúa en un nivel más profundo de conciencia que tiene que ver con el componente espiritual.

Tal y como lo explica Gallegos (1997), el amor universal representa la expansión de nuestra conciencia, la compenetración con el Universo del cual formamos parte, involucra un sentimiento de religación con nuestra esencia y con nuestra Madre Tierra (Gaia), es una visión holística.

Para Gallegos (1999):

[...] la visión holística es el "supuesto de totalidad", que percibe que en el universo todo está conectado con lo demás, es una red viva de relaciones constituida por totalidades/ parte, todo está interconectado formando sistemas y subsistemas; el ser humano es uno con este universo, su conciencia es la conciencia del universo. (p. 7) 
Es necesaria esa claridad para el entendimiento del amor como vida y de la educación como un acto de amor, que involucra la comprensión de la existencia como un proceso armónico y a la vez, caótico. Es preciso penetrar en el eslabón místico que entraña la conexión oculta entre materia y energía; una danza sin fin que se transforma, y da origen a formas genuinas de supervivencia y de convivencia; el denominado "flujo universal" que señala Bohm (1995) en su teoría del orden implicado, en la que explica las interacciones sutiles en todo cuanto existe.

Es así, como EL AMOR y EL CAOS, no pueden ser vistos como elementos contrapuestos o desligados desde la educación, como se han tratado comúnmente; son elementos constitutivos de la creación y parte inherente del desarrollo de nuestras sociedades.

Para Eisler (1998), "El amor se reconoce como la máxima expresión de la evolución de la vida en el planeta, así como el poder unificador universal" (p. 166). Según Cosachov (2000), "El amor es un estado en el que las sensaciones son invasoras, es búsqueda y reclamo. "Es sustancia sagrada" (p. 13).

El caos, por su parte, representa la simplicidad y la complejidad, a la vez; se vislumbra como desorden y orden, como desequilibrio y autopoiésis (palabra que viene de los vocablos griegos autos, que indica sí mismo, y poiesis, que quiere decir producir). De acuerdo con Maturana (1999), todos los sistemas vivos, son sistemas autopoiéticos, se reproducen a sí mismos, continuamente, es decir, son redes de producciones. Este proceso creativo, lleva implícito el ritmo del cambio, la renovación y la vida.

Briggs y Peat (1999) señalan que:

El término científico "caos", se refiere a una interconexión subyacente que se manifiesta en acontecimientos aparentemente aleatorios. "La ciencia del caos se centra en los modelos ocultos, en los matices, en la "sensibilidad" de las cosas y en las "reglas" sobre cómo lo impredecible conduce a lo nuevo [...] el caos es, al tiempo, muerte y nacimiento, destrucción y creación. (pp. 4 y 5)

En este sentido, educar tal y como lo afirma Rodríguez (1997), sería cultivar, armoniosamente, cuerpo, mente, capacidades técnicas, sentido artístico y orientación ética. La educación, se constituye en un proceso de convivencia y coevolución que trasciende el espacio del aula y de la institución, es búsqueda de sentidos compartidos, interconexión, descubrimiento, creación e innovación.

Si reconocemos que el ser humano está ligado al cordón umbilical universal que lo conecta e integra todo, la Universidad, en sus ofertas académicas, tendría que pensar en alternativas educativas ajustadas a nuevas realidades. Es relevante que repiense su papel y el tipo de capacidades que tendría que estar fomentando en los seres humanos, de cara a las nuevas exigencias, las cuales sobrepasan el ámbito del conocimiento técnico y disciplinar.

Ciertamente las universidades hoy se encuentran en un momento de incertidumbre y por qué no decirlo, de crisis, la cual desde una comprensión de la complejidad, tiene que comprenderse como oportunidad para la renovación. Es necesario reconocer, que las fluctuaciones en todos los ámbitos de la vida son perennes, por lo tanto, tenemos que propiciar el desarrollo de la inteligencia, fundada en la capacidad de asumir lo incierto.

Briggs y Peat (1999), mencionan que:

[...] cada uno de nosotros, como individuos, está interconectado con los sistemas de la naturaleza, de la sociedad y del pensamiento que nos rodean y que fluyen a través de nosotros. Vivimos dentro movimientos que afectan a los demás, como los de los demás nos afectan a nosotros, y todo ello crea un caos imprevisible a muchos niveles. (p. 7) 
La trama de la vida es, sin duda, sorprendente, y desde la educación se debe aprender a comprender ese principio de interdependencia que forma parte del perfeccionamiento humano, de ahí se desprende la conclusión de que no hay límites entre el Universo y el ser humano; por el contrario: existe una unión trascendental, que permite la interacción e integración de todo cuanto existe; cuando se logra esto, la vida recobra sentido. Al respecto, Leonard (1987), señala que:

Por muchas que sean nuestras imperfecciones, en el fondo de todos nosotros existe un pulso silencioso de ritmo perfecto, un complejo de formas de ondas y resonancias, absolutamente individual y único, y que, sin embargo, nos conecta con todas las demás cosas del universo. El acto de ponernos en contacto con este pulso puede transformar nuestra experiencia personal y, de un modo u otro, alterar el mundo que nos rodea. (p. 14)

Como se ha señalado, los hilos que nos unen con el universo son sutiles, casi invisibles, sus conexiones secretas representan aún un enigma, a pesar de las múltiples investigaciones que se hacen al respecto. Lo que sí se tiene claro, es que nuestra existencia está, inexorablemente, vinculada al parto cósmico de la creación, existimos en complementariedad. Nuestra constitución vincula, de manera creativa y artística, dos fuerzas polares bajo las cuales, se da el equilibrio perfecto: el Yin y el Yan.

Según Porket (citado por Capra, 1982), el Yin representa la sensibilidad, todo aquello que sea contractivo, conservador; mientras que el Yan involucra lo agresivo, expansivo y exigente. Estos dos polos, en su sentido original, se integran en nosotros. "[...] la personalidad de un ser humano no es una entidad estática, sino un fenómeno dinámico, originado por la interacción entre sus elementos [...]" (p. 39). Esta idea es relevante en función de que representa otro desafío para la educación y sus propulsores: el considerar y aceptar que coexistimos en equilibrio dinámico.

Según Morin (2003):

Somos producto/productores de una auto-eco-re-organización, de donde ha emergido y se ha desarrollado la trinidad humana en la que somos, en tanto individuos productos y productores [...] A la manera de un punto del holograma llevamos en el seno de nuestra singularidad no sólo toda la humanidad, toda la vida, sino casi todo el cosmos, incluido su misterio que yace en el fondo de nuestros seres. (p. 55)

Podemos afirmar que, desde los sistemas educativos, se tiene que promover el amor por la vida. Todo ser viviente tiene un propósito y una función dentro de la danza cósmica de la creación. La educación tiene como papel el lograr que cada persona autodescubra su potencial creador, mediante procesos de mediación pedagógica enfocados al encuentro de sentido, a la conexión con la vida y a su entendimiento como un milagro y no una casualidad, y que entienda que nada de lo que ocurre es producto del azar.

Lo anterior implica destituir la noción antropocéntrica, en la que se visualiza al ser humano, como superior permitiéndose, en mayor o menor medida, la explotación y esclavitud de otros seres humanos y de múltiples seres vivos.

La educación superior tiene que instruir más a la persona en el conocimiento de su potencial, para que cuide y proteja a los demás seres, para que asuma el compromiso con la vida mediante una actuación respetuosa y dignificadora, apoyado y aportando su parte al principio de la sincronía y amor universal.

Si deseamos que la educación tenga impacto real en el desarrollo de la persona y, por ende, del planeta y del Universo, será, ante todo, necesario, aprender a danzar con el paradigma de la complejidad, la incertidumbre y el caos. Es urgente que ampliemos nuestros horizontes. 
No podemos seguir fijando tiempos, espacios, conocimientos, valores. ¿Qué ocurre con el afecto, la ternura, la pasión?; ¿No son acaso éstas las fuerzas más poderosas para llenar de significado la vida de una persona?

Si hoy reconocemos que el pensar y el sentir son atributos de la inteligencia, es contradictorio seguir educando con la linealidad con que se han concebido los procesos de conocimiento y de aprendizaje. Como lo afirma Assman (2002): "Hoy día sabemos [...] que aprender no se puede reducir a una apropiación de los saberes acumulados por la humanidad. Se aprende no sólo con el cerebro ni sólo en la escuela" (p. 27).

Lo que se aprende está ligado, entonces, no a los contenidos, sino a los sentidos que le encontramos a ellos, a la posibilidad de aplicación, de crecimiento, de conciliación; de placer; lo cual conlleva implícitamente, procesos complejos de autoidentificación. No se puede aprender aquello que no se siente ni se vive. La educación no debe tener escalas de tiempos yde espacios; debe convertirse en una puerta abierta a la creatividad para la libertad.

El aprendizaje como proceso vital, tiene, necesariamente, que ser creativo; y lo creativo, está ligado a la construcción de sentidos y significativos, lo que se convierte, en última instancia, en una aventura constante de transformación. Según Leonard (1987), el cerebro tiene una capacidad creadora infinita. Esta premisa básica, debe ser parte de la acción formadora de la persona, pues toda experiencia pedagógica, debe estar cargada de sensibilidad.

De ahí que el aprendizaje no debe fragmentarse o desvincularse de la vida. Las prácticas curriculares de fragmentación, entre las ciencias sociales y las ciencias naturales, es teoría que ha dejado heridas profundas en muchas mentes y corazones.

La Universidad debe ir en búsqueda de una educación holística, que unifique e integre los conceptos, las nociones, los espacios y los tiempos. El despertar del interés por el aprendizaje, se obtiene sólo cuando se logra enganchar la cotidianidad de la vida. Ese vínculo proporciona posibilidades de originalidad, lo que permite que la mente se libere, y se favorezca el fluir de la energía vital.

Hablar de sentir, sin duda, involucra comprender que allí se encuentra el núcleo, la fuerza que dispara la creatividad y origina el aprendizaje, implica reconocer, que el todo está en el todo, que es circularidad, participación, movimiento, la dualidad, el encuentro el punto de unión de la conciencia.

La creatividad puede entenderse como armonía del orden natural, expresa en todos los seres que habitan el planeta y, por tanto, en las fuerzas que rigen el Universo.

Al respecto, Bohm (2002), expresa que "[...] las manifestaciones de la creatividad en la humanidad no son sólo similares a los procesos creativos de la naturaleza, sino que son del mismo carácter intrínseco que las fuerzas creativas del universo" (p. 7).

Por tales razones, si se logra que la educación se sumerja en las redes de la creatividad, probablemente, encontrarán niveles de mayor productividad humana en todos los órdenes de la vida. Ese sentido de lo ético y lo estético, puede provocar los cambios tan deseados para consolidar la visión de ciudadanía planetaria.

La educación superior tendrá, entonces, que poner más atención para crear ambientes y procesos creadores, que impacten la vida social, económica, política y cultural. Incluir la creatividad en los procesos universitarios, sin duda, incentivaría el interés por aprender, porque cuando se hace y rehace la cultura y la vida teniendo protagonismo, la educación se convierte en un proceso placentero y transformador.

Prado y Gutiérrez (2004), hablan de la Pedagogía del Lenguaje Total y argumentan que es una síntesis pedagógica que, con base en una nueva visión del hombre y de la sociedad, concibe 
el aprendizaje como un proceso endógeno y permanente, que hace que el educando sea creador, actualizador y realizador de su propio ser [...] (p. 21).

Inmersos en la colectividad, tenemos que aprender a estremecernos con los diversos lenguajes, eso forma parte de la esencia de una nueva cultura planetaria. Cuando nos demos cuenta del significado de nuestras opiniones, seremos capaces de compartir un contenido común y es que formamos parte de una red de comunicación compleja.

El objetivo de la comunicación o del diálogo, no es analizar las cosas, imponer un argumento o modificar las acciones de los demás, es como expresa Bohm (2001) "[...] suspender las propias creencias y observarlas, escuchar todas las opiniones, ponerlas en suspenso y darnos cuenta de su significado" ( $\mathrm{p}$.55).

El diálogo genera lazos de esperanza, ofrece espacios para ensayar soluciones para los viejos y nuevos problemas que enfrenta el planeta. No se trata, únicamente, de resolver las dificultades de una sociedad, se trata, ante todo, de reconocerse en el diálogo y transformarse en éste, el cual forma parte de la conciencia colectiva e individual que se transfigura en conocimiento.

El conocimiento es un proceso que, en la puesta en práctica, no debe manejarse como el conjunto fijo de verdades, ya que éste es siempre cambiante. Gutiérrez (citado en Gutiérrez y Prieto 2004) considera que, "admitimos conocimientos cada vez que observamos una conducta efectiva (o adecuada) en un contexto señalado, es decir, en un dominio que definimos con una pregunta (explícita o implícita) que formulamos como observadores" (p. 12).

Cada fase de la vida, tiene memoria activa y ésta goza de una esencia, que es el pensamiento, el cual incluye una respuesta intelectual, emocional, sensitiva, muscular y física. Este proceso es constante, no puede mecanizarse, la desarticulación genera confusión, lo que puede llevar, a su vez, a la destrucción.

La creatividad está asociada al conocimiento y "[...] conocer es un arte, un impulso del cual surge el "amor por la sabiduría" (Bohm, 2002, p. 133) que se construye cuando se es capaz de articular el todo con todo, de forma que permita el fluir para germinar el desarrollo del pensamiento complejo, relacionado con la capacidad de aprendiencia.

Como bien sabemos, el ser humano, está siempre en un constante aprender, es un proceso orgánico activo, que tendría que ser, por tanto, como antes se mencionó: gozoso, para entrar en contacto. Según Assman (2002), la APRENDIENCIA implica la unidad entre los procesos cognitivos y los procesos vitales. Para llegar a la verdadera aprendiencia tenemos que encontrar el placer y el afecto, Bach (2004) plantea la importancia de que nuestro pensamiento y espíritu se mantenga siempre inquieto y dispuesto a aprender y ese sentido nos recomienda: "La criatura estudiosa que llevas adentro, el inquieto ser espiritual que encarna tu auténtica personalidad, te guía por la vida. No vuelvas la espalda a los futuros posibles, antes de estar seguro de que no tienes nada que aprender de ellos" (p. 6).

Para las biociencias, tanto los procesos de la vida como los de aprendizaje son en el fondo, una misma cosa. Entre la vida y el aprendizaje, existe una unidad, ésta es la aprendiencia, según Boff (1996), "los frutos de la educación no se pueden resumir ya en conocimientos acumulados" (p.16), sino en la capacidad para arriesgarse y proponer transformaciones que nacen de su autoconciencia sobre su esencia social y universal.

La humanidad está experimentando una transición que trastoca todas las estructuras del conocimiento, dada la complejidad de la realidad en la que convive; una sociedad basada en las nuevas tecnologías de la información y el conocimiento, la globalización de las economías mundiales, la interculturalidad que nos lleva, indudablemente, a aceptar que vivimos en una era planetaria que exige de una comprensión profunda de la vida. En opinión de Assmann (2002), '’[...] la sociedad 
entera, debe entrar en un estado de aprendizaje y transformarse en una misma red de ecologías cognitivas" (p. 19).

Los seres humanos, deben actuar con la responsabilidad y la libertad que se le otorga como organismo viviente, como parte de esa comunidad planetaria según Maturana (1989), hay que fortalecer la conciencia, [...] permitir y facilitar el crecimiento [...] como seres humanos que se respetan a sí mismos y a los otros con conciencia social y ecológica, de modo que puedan actuar con responsabilidad y libertad en la comunidad a que pertenecen a través de la comunicación" (p. 25).

Es importante tomar en cuenta que, actualmente, la educación no sólo es fundamental para la calidad de empleo, sino para la calidad de vida. La funcionalidad educativa se valora en términos de desarrollo humano social sostenible, aspecto que sobrepasa la simple empleabilidad.

Es fundamental seguir trabajando desde la educación universitaria, la mente y el corazón, porque tal y como lo indica Assmann (2002): '’[...] es preciso trabajar pedagógicamente el desequilibrio de los seres humanos, en relación con las oportunidades contenidas en las obras de sus propias manos. El atraso ha pasado a ser, sobre todo, de las mentes y de los corazones" (p. 21). No se puede obviar, que en la calidad educativa, necesariamente, se vinculan aspectos pedagógicos y emotivos.

Tanto el concepto de vida, como el de conocimiento, deben ser redefinidos, y la Universidad, tiene ese desafío, la articulación entre procesos cognitivos entendidos como procesos vitales.

Desde la pedagogía universitaria, se tienen que generar, intencionalmente, experiencias de auténtico aprendizaje, debe darse un "ambvientre", palabra construida a partir de la unión de ambiente más vientre, entendido como un estado de conciencia profunda, en el que se alcanza dar ese salto cuántico, que posibilita la creación y la recreación que trasciende el tiempo y el espacio.

Es importante, también, volver a introducir en la Universidad, el concepto de MORFOGÉNESIS del conocimiento, que según Assmann (2002): “[...] implica, además de la instrucción informativa, la reinvención y construcción personalizada del conocimiento" (p. 29).

Si esta perspectiva es tomada en cuenta para el enriquecimiento pedagógico, se deben considerar, entonces, algunos principios como los siguientes:

- $\quad$ Aprender es un proceso creativo que se autoorganiza.

- Todo conocimiento tiene una inscripción corporal del conocimiento.

- La dinámica de la vida y del conocimiento están unidas.

- $\quad$ El placer es dinamizador del conocimiento.

- Urge flexibilizar los lenguajes pedagógicos.

Cabe recordar lo que plantea Assmann (2002), 'El mundo se está transformando en una trama compleja de sistemas aprendientes"' (p. 21). Si el cerebro es concebido como un órgano social por excelencia, es preciso reconocer que los sentidos se construyen a partir de la vivencia cultural, en permanente interacción con el medio ambiente y el lenguaje.

La Universidad debe ser propiciadora, sin duda, de experiencias plurivalentes de aprendizaje, capaces de mirar la riqueza que contempla la diversidad como forma de vida. Tiene que permitir un contexto y un ambiente adecuado para iniciar al estudiante, en vivencias personalizadas de aprender a aprender. Esto se puede lograr con la flexibilidad, no sólo del proceso del conocimiento, sino de las experiencias de aprendizaje, que éste pueda y deba generar. Como lo expresa Osho (1999), para fluir en forma armónica en la vida se debe tener presente que el ser humano es parte de una esencia creativa que produce en tanto se permita la expresión. 
El mejor proceso educativo es aquel en el que se da la interacción entre el cerebro y los sentidos, que es lo que conduce, de una u otra forma, a la esencia del conocimiento, y provoca el "aprendizaje con sentido".

La educación superior, tiene que reinventarse, construirse, reconstruirse mediante experiencias en las que el intercambio de saberes y emociones sean parte de la cotidianidad. Morin (2003), menciona que en la educación del futuro: "La religazón debe sustituir a la dispersión y recurrir a la "simbiosofía", esto es, la sabiduría de vivir unidos" (p. 13). Agrega que la esencia de la persona necesita estar acompañada, nutrida y educada, por siete saberes que desarrollan la conciencia en planos superiores. Todos ellos conectados entre sí y son: ser, vivir, crear, conocer, dialogar, responder y amar. Entre todos éstos, hay un delgado hilo energético, que los une y mantiene en interacción.

Para concluir, podemos decir, que el amor, el caos y la creatividad, tienen que estar presentes en todo proceso educativo, para provocar aprendizajes, y que tanto las estructuras intelectuales de los individuos como las sociales, pasan por esos procesos.

La vida y el conocimiento están unidos; es por este motivo que el aprender a vivir, es aprender a encontrar la relación entre todos estos saberes y la comunión con los seres, sin olvidar la simbiosofía, la cual se desarrolla en el $A M O R$ y por el $A M O R$.

Es importante resaltar que la visión holística de la educación de la cual hemos estado hablando en este ensayo, tiene sus esencias en la física cuántica, visión que supera el dogmatismo, aspecto que aún hoy encuentra gran resistencia, porque es una transformación de conciencia, de pertenencia a un todo integrado. El Universo toma un nuevo matiz, porque se reconoce que tanto la ciencia como la espiritualidad, facilitan la amplitud del conocimiento y expanden la conciencia, aspecto que permite la caída de un paradigma esclavizador.

\section{REFERENCIAS BIBLIOGRÁFICAS}

Assman, H. (2002). Placer y ternura en Educación. Hacia una sociedad aprendiente. Madrid, España: Narcea Ediciones.

Bach, R. (2004). Mensajes para siempre. Buenos Aires, Argentina: Vergara \& Riba Editoras.

Boff, L. (1996). Ecología: grito de la tierra, grito de los pobres. Madrid, España: Editorial Trotta.

Boff, L. (2002). Espiritualidad: un camino de transformación. Santander, España: Salterrae.

Bohm, D. (1995). Teoría del orden implicado. Barcelona, España: Editorial Kairós.

Bohm, D. (2001). Sobre el diálogo. Barcelona, España: Editorial Kairós.

Bohm, D. (2002). La totalidad y el orden implicado. Barcelona, España: Editorial Kairós.

Briggs, J. \& Peat, D. (1999). Las siete Leyes del Caos. Las ventajas de una vida caótica. Barcelona, España: Revelaciones.

Capra, F. (1982): El Punto Crucial. Buenos Aires, Argentina: Troquel. 
Cosachov, M. (2000). Entre el cielo y la tierra. Un viaje por el mapa del conocimiento. Buenos Aires, Argentina: Biblos.

Eisler, R. (1998). Placer sagrado: Sexo, Mitos, y Política del cuerpo. Santiago, Chile: Editorial Cuatro vientos.

Gallegos, R. (1997). Una sola conciencia. Enfoque holístico sobre el futuro de la humanidad. México: Editorial Pax.

Gallegos, R. (1999). Educación holística. Pedagogía del amor universal. México: Editorial Pax.

Gutiérrez, F. \& Prieto, D. (2004). Mediación pedagógica. Guatemala: Universidad San Carlos de Guatemala.

Leonard, G. (1987). El pulso silencioso. Búsqueda del ritmo perfecto que existe dentro de nosotros. Madrid, España: Edaf.

Maturana, H. (1989). Emociones y lenguaje en educación y política. Santiago, Chile: Dolmen Ediciones.

Maturana, H. (1999). Transformación en la convivencia. Santiago, Chile: Dolmen Ediciones.

Morin, E. (2003). Método V. La humanidad de la humanidad. La identidad humana. Madrid, España: Cátedra Teorema.

Prado, C. \& Gutiérrez, F. (2004). Germinando Humanidad. Pedagogía del aprendizaje. Guatemala: Save the Children-Noruega.

Rodríguez, R. (1997). Del Universo al Ser Humano: Hacia una concepción planetaria para el siglo XXI. Madrid, España: McGraw-Hill.

Shree, B. (Osho). (1999). Meditación: una introducción a la comprensión contemporánea de la meditación. Barcelona, España: Editorial Debate. 